Research Article

\title{
Experiment and Theoretical Analysis Study of ETFE Inflatable Tubes
}

\author{
YanLi He ${ }^{1,2}$ and WuJun Chen ${ }^{1}$ \\ ${ }^{1}$ School of Naval Architecture, Ocean \& Civil Engineering, Shanghai Jiao Tong University, Shanghai 200240, China \\ ${ }^{2}$ State Key Laboratory of Ocean Engineering, Shanghai Jiao Tong University, Shanghai 200240, China \\ Correspondence should be addressed to YanLi He; yanlihe@126.com
}

Received 10 August 2014; Revised 21 October 2014; Accepted 22 October 2014; Published 11 November 2014

Academic Editor: Hong Nie

Copyright (C) 2014 Y. He and W. Chen. This is an open access article distributed under the Creative Commons Attribution License, which permits unrestricted use, distribution, and reproduction in any medium, provided the original work is properly cited.

The load bearing capacity of an ETFE (ethylene-tetra-fluoro-ethylene) inflatable tube is tested in this paper, and a comparative study of two wrinkling theories, the bifurcation theory and the tension field theory, is carried out for wrinkling analysis of the ETFE inflatable tube. Results obtained from the bifurcation theory and experiment reveal the limitations of tension field theory on the wrinkling analysis. The load-displacement curves of inflatable beams under bending load are obtained and compared with the experimental results; curves obtained using the bifurcation theory show coincidence with experimental curves, but the curves obtained using the tension field theory have noticeable deviations between calculated and experimental results.

\section{Introduction}

Inflatable membrane structures will be widely used in future space missions, because they are lightweight and easy to fold. But a membrane element has negligible bending stiffness; the wrinkles are easy to emerge during manufacture and packaging, folding, and compression of these membrane structures. The presence of wrinkles in membranes is a main cause of surface errors and reducing load bearing capability of precision space membrane structures. Such errors restrict their technical function performance, for that reason, many studies on wrinkling phenomena for these structures are carried out.

Many researchers have developed methods for conducting the wrinkling analysis of membrane structures. One of the earliest works is the tension field theory, in which it is assumed that the membrane has no bending stiffness and can carry no compressive stress. From these assumptions, wrinkling of initially flat membranes is treated as in-plane problems through the special definition. Therefore, in tension field, only wrinkled regions and directions of wrinkles are obtained. Mansfield $[1,2]$ developed the famous tension field theory and did analysis of wrinkled membrane's load transfer. Pipkin [3] studied the relaxed energy density for isotropic elastic membrane. The first finite element solution to incorporate wrinkling theory was the iterative materials properties model (IMP) developed by Miller and Hedgepeth [4] and Miller et al. [5]. It is based on the observation that if during a simulation a membrane element is deemed to be wrinkled, the geometric strain in the direction perpendicular to the direction of the wrinkles, due to out-of-plane deformation of the material, can be modeled by introducing a variable effective Poisson's ratio for the element. By applying the iterative membrane properties method, Adler [6] implemented this model as a user-defined material (UMAT) subroutine in the ABAQUS finite element package. Yang et al. [7] presented an analysis procedure of the wrinkling of membrane structures with the modified constitutive relationship method; an integrated algorithm is developed, in which the Newton-Raphson method and updated Lagrange formulation are adopted. With the proposed algorithm, two fabric roof structures are analyzed; results show that the presented method is feasible and reliable.

In actual situations, wrinkling phenomena are due to bifurcation and are intensely affected by small bending stiffness; Wong et al. proposed another geometrically nonlinear analysis method for wrinkling phenomena based on the 
bifurcation theory. In bifurcation theory, detailed wrinkling behaviors, such as number and amplitude of wrinkles, can be obtained, while in tension field theory this kind of information cannot be obtained. Tomita and Shindo [8] were the first to make use of a three-dimensional shell description of a wrinkled membrane, in a study of the residual wrinkles in a thin metallic plate that has been pulled diagonally. Wong and Pellegrino [9-11] gave a detailed experimental study of the evolution and shape of reversible wrinkles. He proposed a general analytical model to determine the location and pattern of wrinkles in thin membranes and to estimate their wavelength and amplitude. On the basis of this, geometrically nonlinear finite element models of membrane structures were used to simulate the onset and growth of wrinkles. Jarasjarungkiat et al. [12] gave a systematic verification of a wrinkling model based on the material modification approach by means of an analogy between wrinkling and plasticity. Both isotropic and orthotropic materials were studied. Jarasjarungkiat et al. [13] employed the projection method from the inelastic model to take into account wrinkling of thin membranes, and the wrinkling strain component was removed from the total strains. Wang et al. [14] presented a novel shell-membrane concept to define the wrinkling analytical object, an analytical model based on the bifurcation theory of thin-plate is introduced to predict the wrinkling wavelength and amplitude, numerical simulation incorporating nonlinear postwrinkling analysis is employed to simulate the detailed nonlinear wrinkling behavior, and results from numerical simulation show good agreement with the analytical prediction. Tan et al. [15] presented the definition of dimensionless bending stiffness factor, the relationship of bending stiffness factor and wrinkling factor is derived, and the bending stiffness factor is simplified as different linear functions with wrinkling factor. The bending stiffness including expression of wrinkling factor is substituted into the deflection differential equation, and then the slope and deflection equation of the inflatable beam is deduced by integrating the deflection differential equation. Nguyen et al. [16] analyzed the linear eigen and nonlinear buckling analysis of an inflatable beam made of orthotropic technical textiles. The nonlinear finite element solutions were investigated by using the straightforward Newton iteration with the adaptive load stepping for tracing the load-deflection response of the beam. To assess the effect of geometric nonlinearities and the inflation pressure on the stability behavior of inflatable beam, a simply supported beam was studied. The influence of the beam aspect ratios on the buckling load coefficient was also pointed out. Xiao [17] set up a numerical procedure for membrane wrinkle analysis and used it to analyze a square planar film reflectarray which was under pure shear force and to evaluate its induced wrinkle characteristics.

In this paper, a comparative study on wrinkling analysis using engineering elastic beam theory, tension field theory, and bifurcation theory is carried out, the wrinkling of an ETFE inflatable membrane tube is investigated, and, at the same time, the experiment of the ETFE inflatable tube is conducted to verify the numerical analysis results.

\section{Experiment of Load Bearing Capacity of an Inflatable Tube}

A full-scale ETFE inflatable beam was fabricated and the deflections at three points under bending are measured; the layouts of the test rig and the measuring points are shown in Figure 1. The cantilever tube is fixed on a T shape steel frame, the dimensions of the model are $2533 \mathrm{~mm}$ in length and $426 \mathrm{~mm}$ in diameter, and the thickness of ETFE is $250 \mu \mathrm{m}$. The test model of the ETFE inflatable tube under bending loads is shown in Figure 2, and the wrinkles in wrinkled regions of the inflatable tube are shown in Figure 3.

The experimental load-deflection curves of three points at internal pressure values of $3 \mathrm{kPa}, 4 \mathrm{KPa}$, and $5 \mathrm{kPa}$ are shown in Figures 4(a), 4(b), and 4(c), respectively. Seen from Figure 4, when internal pressure is $3 \mathrm{Kpa}, 4 \mathrm{Kpa}$, and $5 \mathrm{Kpa}$, the critical load bearing capacity of the inflatable tube is $51.9 \mathrm{~N}, 72.5 \mathrm{~N}$, and $90.2 \mathrm{~N}$, respectively.

\section{Theoretical Load Bearing Capacity of Inflatable Tube}

The engineering elastic beam theory is often used during the preliminary design of inflatable structures. Based on the engineering elastic beam theory, the internal pressure is assumed to sustain the section configuration, the rotation axis of section of balanced moment is still passing through the center of the section, the stress of thin films is zero in the wrinkled region along the wrinkling direction, and then the relationship between wrinkling angle and load is obtained by force and bending moment equilibrium equations of the section. The analysis model of the inflatable cantilever tube is shown in Figure 5, which is similar to traditional EulerBernoulli beam theory, but it introduces the influence of membrane wrinkling.

For a thin-walled tube, the longitudinal stress and hoop stress can be expressed as $[18,19]$

$$
\begin{aligned}
\sigma_{c} & =p R \\
\sigma_{l} & =\frac{1}{2} p R,
\end{aligned}
$$

where $\sigma_{c}$ is hoop stress of inflatable tube and $\sigma_{l}$ is longitudinal stress of inflatable tube.

As shown in Figure 5, the moment of inertia in the wrinkled region of the beam is as follows [20]:

$$
\begin{aligned}
& I=r^{3} t[\pi-\theta+\sin \theta \cos \theta] ; \quad\left(\frac{\pi p r^{3}}{2}<M<\pi p r^{3}\right), \\
& I=\pi r^{3} t \quad\left(M<\frac{\pi p r^{3}}{2}\right) .
\end{aligned}
$$

Here, $p$ is internal pressure, $r$ is radius of cross section, and $t$ is thickness of membrane.

The relationship between moment and wrinkling angle in wrinkled region can be expressed in the following form [20]:

$$
\frac{F \cdot x}{p r^{3}}=\frac{\pi(2 \pi-2 \theta+\sin 2 \theta)}{4[(\pi-\theta) \cos \theta+\sin \theta]} \quad(0<\theta<\pi) .
$$




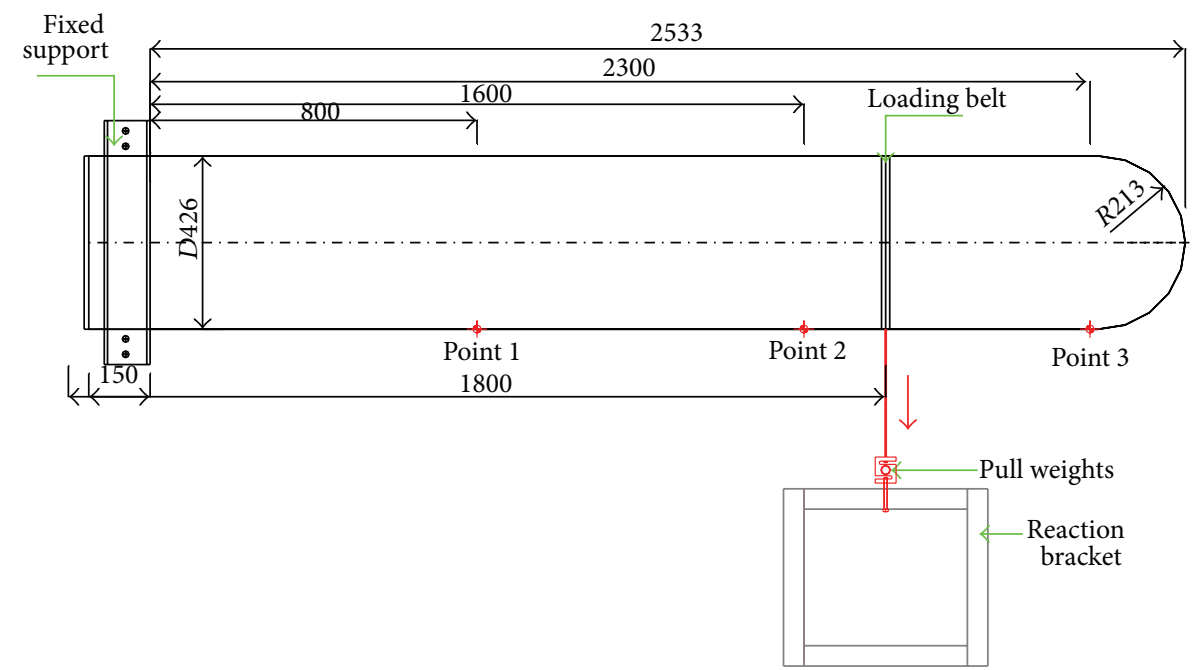

(a) Top view

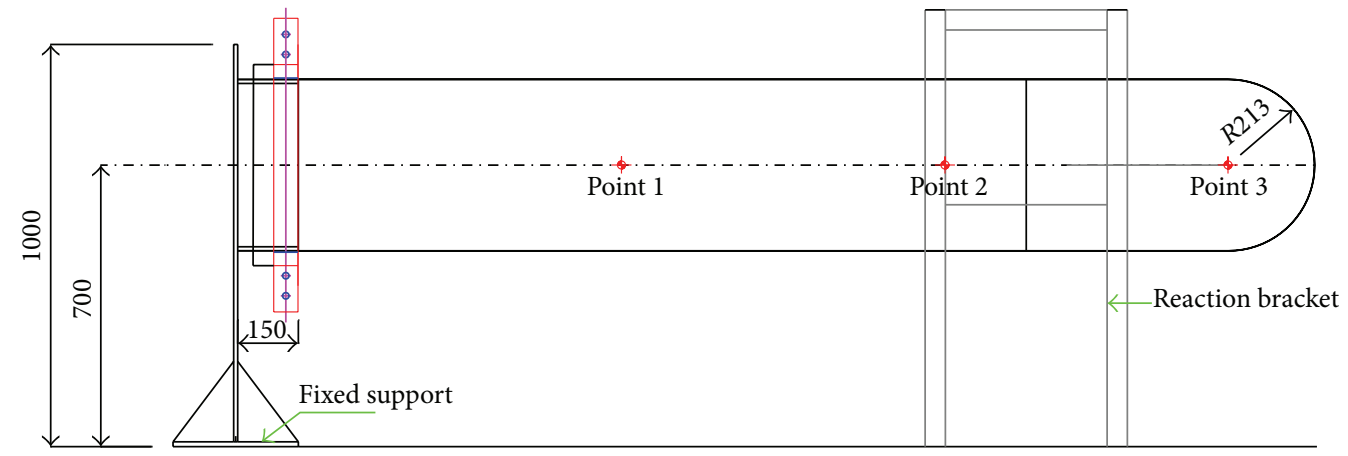

(b) Side elevation view

Figure 1: Layout of measuring points and test rig.

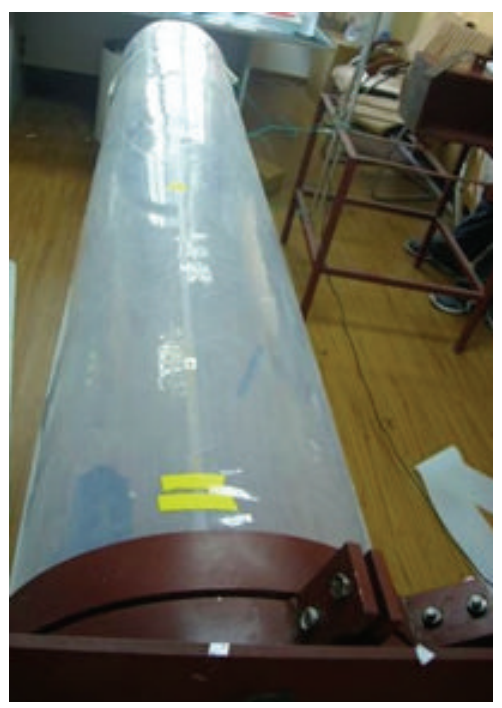

FIGURE 2: The test model of an ETFE inflatable tube.

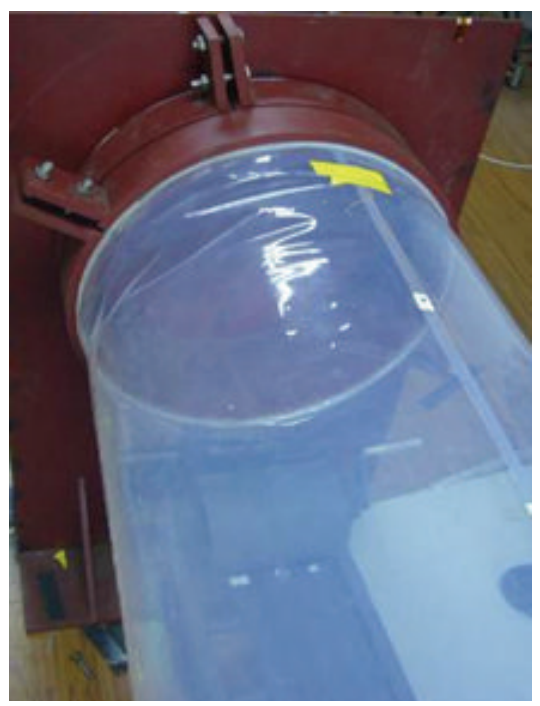

FIGURE 3: Wrinkles in wrinkled region. 


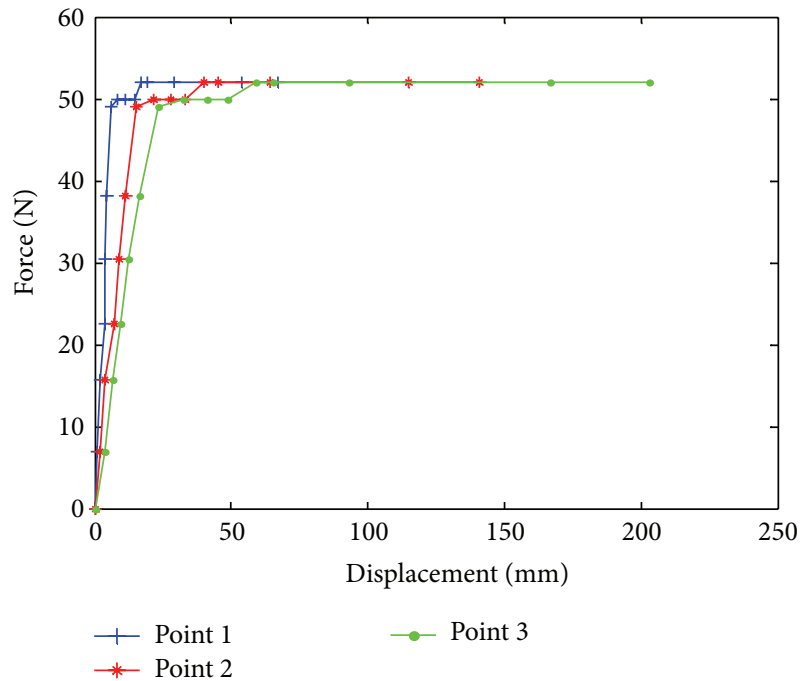

(a) Internal pressure $=3000 \mathrm{~Pa}$

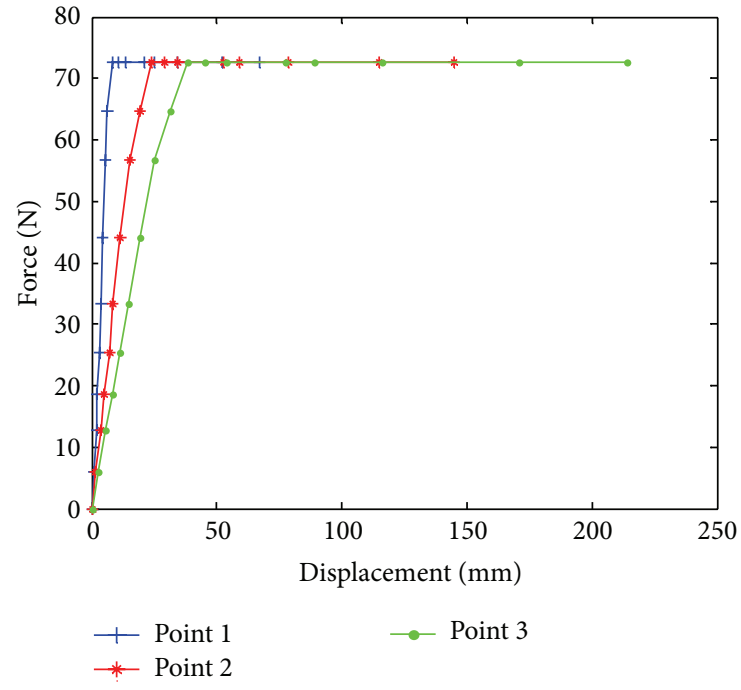

(b) Internal pressure $=4000 \mathrm{~Pa}$

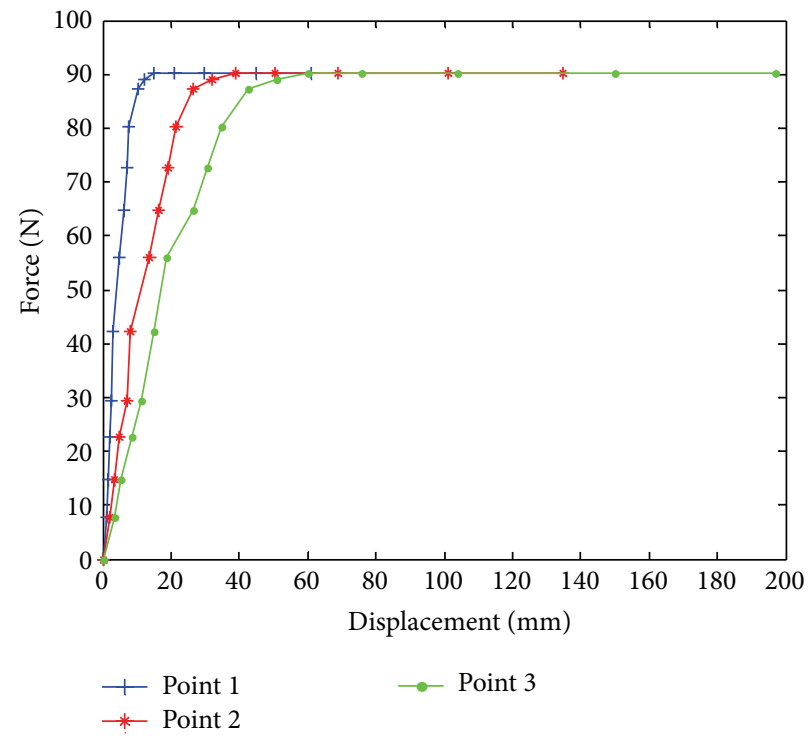

(c) Internal pressure $=5000 \mathrm{~Pa}$

FIGURE 4: Experimental load-deflection behavior of the ETFE inflatable tube.

Based on the membrane theory, failure occurs when the wrinkles propagate completely around the circumference; from (3), the failure moment is expressed as [21]

$$
M_{u}=\pi p r^{3} .
$$

Then, the critical force is expressed as

$$
F_{u}=\frac{M_{u}}{l}
$$

where $l$ is the distance between the fixed support and loading belt.

According to (4), the critical bearing capacity can be easily obtained, the results are shown in Table 1 , and the errors between the experimental data and results of engineering elastic beam are also shown in Table 1.

Seen from Table 1, the theoretical critical bearing capacities obtained from (4) are all less than experimental results; the errors between them are about $5 \%$ to $7 \%$.

\section{Analytical Approaches: Tension Field Theory}

4.1. Wrinkling Criteria. In general, there are three criteria for predicting the occurrence of the membrane wrinkling, the principal stress criterion, the principal strain criterion, and the principal stress-strain criterion. The tension field theory 

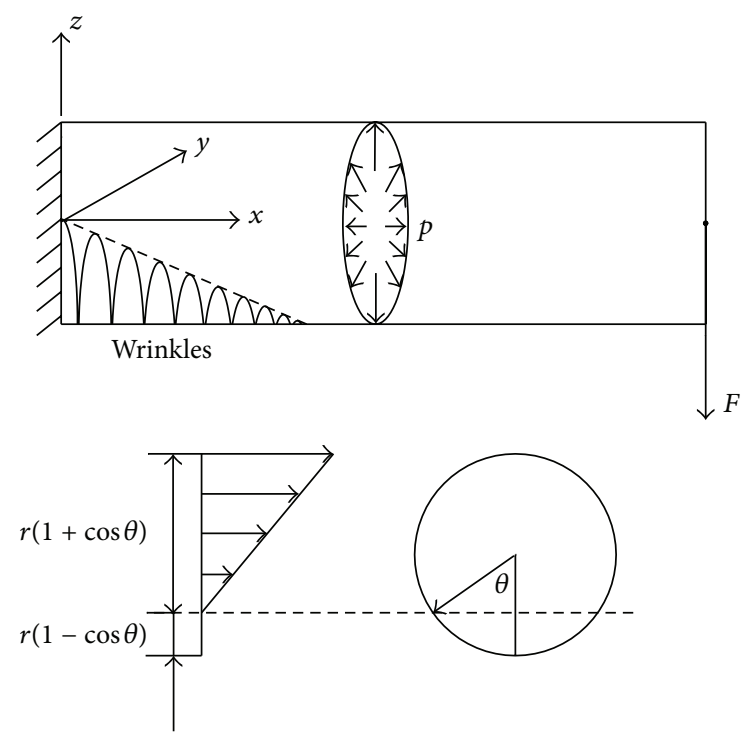

FIGURE 5: Analytic model of the inflatable cantilever tube.

treated in this paper is based on the principal stress-strain criterion, which can be described as

$$
\begin{gathered}
\sigma_{2}>0, \quad \text { Taut } \\
\sigma_{2} \leq 0, \quad \varepsilon_{1}>0, \quad \text { Wrinkled } \\
\sigma_{2} \leq 0, \quad \varepsilon_{1} \leq 0, \quad \text { Slack, }
\end{gathered}
$$

where $\sigma_{2}$ is the second principal stress and $\varepsilon_{1}$ is the first principal strain.

If some membrane elements are slack or wrinkled, the stresses and the constitutive matrix should be modified according to the following procedure.

4.2. Wrinkling Analysis Procedure. The principal stress vector can be expressed as the product of the stiffness matrix and the strain vector. The first finite element solution to incorporate wrinkling theory was the iterative material properties model (IMP) developed by Miller. It is based on the observation that if a membrane element is deemed to be wrinkled, the geometric strain in the direction perpendicular to the direction of the wrinkles, due to out-of-plane deformation of the material, can be modeled by introducing a variable effective Poisson's ratio for the element.

When $\sigma_{2}>0$, using "taut" modulus matrix, based on Hooke's law for plane stress and given by

$$
\mathbf{D}_{t}=\frac{E}{1-v^{2}}\left[\begin{array}{ccc}
1 & \nu & 0 \\
v & 1 & 0 \\
0 & 0 & \frac{(1-v)}{2}
\end{array}\right] .
$$

When $\sigma_{2} \leq 0$ and $\varepsilon_{1}>0$, using "wrinkled" modulus matrix

$$
\mathbf{D}_{w}=\frac{E}{4}\left[\begin{array}{ccc}
2[P+1] & 0 & Q \\
0 & 2[1-P] & Q \\
Q & Q & 1
\end{array}\right],
$$

\begin{tabular}{|c|c|c|c|}
\hline \multirow[b]{2}{*}{ Internal pressure $(\mathrm{Pa})$} & \multicolumn{2}{|c|}{ Critical bearing capacity $(\mathrm{N})$} & \multirow[b]{2}{*}{ Error } \\
\hline & $\begin{array}{l}\text { Engineering } \\
\text { elastic beam }\end{array}$ & Experiment & \\
\hline 3000 & 50.6 & 52.9 & $-4.4 \%$ \\
\hline 4000 & 67.43 & 72.5 & $-7.0 \%$ \\
\hline 5000 & 84.28 & 90.2 & $-6.6 \%$ \\
\hline
\end{tabular}

where $P=\left(\varepsilon_{x}-\varepsilon_{y}\right) /\left(\varepsilon_{1}-\varepsilon_{2}\right), Q=\gamma_{x y} /\left(\varepsilon_{1}-\varepsilon_{2}\right)$.
TABLE 1: Comparison between the results of elastic beam theory and results of experiment.

When $\sigma_{2} \leq 0$ and $\varepsilon_{1} \leq 0$, using "slack" modulus matrix

$$
\mathbf{D}_{s}=0 \text {. }
$$

4.3. Analytical Model. Figure 6 shows the analytical model used for IMP model; the analytical model has the same dimensions as the test model which is shown in Figure 1; same as the test model, three points are also selected to record loaddisplacement curves; positions of three points and mesh of analytical model are also shown in Figure 6. According to the biaxial test of ETFE film $[22,23]$, the properties of the components are shown in Table 2.

4.4. Numerical Simulation and Results. The tension field theory analysis in this study is performed using program ABAQUS membrane elements. However, since ABAQUS does not include the wrinkling analysis capability, the authors have implemented the IMP method developed by Miller and Hedgepeth [4] and Miller et al. [5] to iteratively modify the element stiffness matrix to accommodate structures wrinkle effects using combined stress-strain based wrinkling criteria.

The simulation was conducted at internal pressure values of $3 \mathrm{KPa}, 4 \mathrm{KPa}$, and $5 \mathrm{KPa}$; the relationship between force applied to the inflatable tube and the deflection has been computed at internal pressure values of $3 \mathrm{KPa}, 4 \mathrm{KPa}$, and $5 \mathrm{KPa}$; the results are shown in Figure 7 . Table 3 lists the comparison of critical bearing capacity at internal pressure values of $3 \mathrm{KPa}, 4 \mathrm{KPa}$, and $5 \mathrm{Kpa}$ between experiment and results from IMP model.

Seen from Table 3, the critical bearing capacities of inflatable tube at internal pressure values of $3 \mathrm{KPa}, 4 \mathrm{KPa}$, and $5 \mathrm{Kpa}$ adopting tension field method are all lower than the results from experiment; the errors between them are about $10 \%$ to $14 \%$.

\section{Nonlinear Postbuckling Analysis}

The bending stiffness of a membrane, although small, plays a key role in determining the shape and amplitude of the wrinkles. The nonlinear buckling finite element analysis incorporating thin shell model is used to simulate the detailed wrinkles in the membrane structure. The key difference between the first approach and the other is the additional burden of the initial eigenvalue/eigenvector extraction. ABAQUS offers several shell elements; both S4R5 and S9R5 are thin shell elements with three in-plane translations and two inplane rotation components. They use reduced integration with hourglass control to avoid shear locking. Both elements 


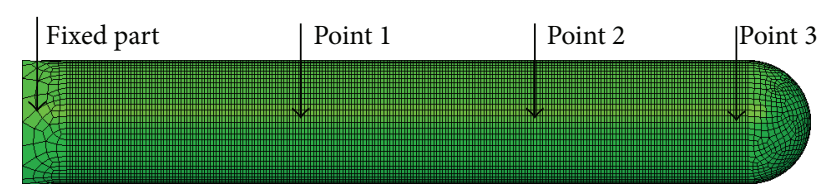

FIgURe 6: Analytical model.

can model thin shells fairly accurately and S4R5 was chosen in this paper since it is computationally more economical.

5.1. Initial Imperfection. Initial imperfection planting in inflatable beam can make the bending analysis more smoothly. An eigenvalue buckling analysis is used to obtain the possible wrinkling modes of the membrane subjected to its actual boundary conditions and loading. A set of eigenmodes has been chosen; geometrical imperfections are introduced in the form of out-of-plane deformations of the membrane, using the * IMPERFECTION directive

$$
\Delta z=\sum_{i} w_{i} \phi_{i}
$$

where $w_{i}$ is the $i$ th eigenmode and $\phi_{i}$ is a scaling factor whose magnitude is chosen as a proportion of the thickness of the membrane. In this paper, $10 \%, 25 \%$, and $100 \%$ of the thickness have been used as $\phi_{i}$, and the sensitivity of the predicted response to different $\phi_{i}$ has been determined; results are shown in Table 4 . Note that the load bearing capacity remains unchanged when the magnitude of the imperfections is changed.

It was thus concluded that the inflatable tube is not sensitive to the size of imperfection. It was decided to use an imperfection consisting of the first ten eigenmodes corresponding to eigenvalues, each multiplied by a scaling factor $\phi_{i}=0.25 t$.

5.2. Sensitivity to Mesh Density. The initial mesh, shown in Figure 6, predicted a smaller number of wrinkles than that observed in the experiment; the longitudinal mesh aspect ratio (longitudinal mesh width/membrane thickness) of the initial mesh is 40 . Therefore, two additional mesh sizes were used to investigate the effect of mesh density on the final load bearing capacity. The longitudinal mesh widths of two new models in the wrinkled region are a half and a quarter of the longitudinal mesh width of the initial mesh, respectively, and mesh width in hoop direction remains unchanged. So, the longitudinal mesh aspect ratios of two new models are 20 and 10 , respectively. The mesh and wrinkles of three models are all shown in Figure 8. Both of the denser meshes predicted the same number of wrinkles as in the experiment shown in Figure 3. Table 5 shows that both of the denser meshes predicted the same load bearing capacity, which suggests that the solution becomes mesh-independent after a particular level of refinement. Therefore, the mesh with four times the number of the initial mesh, shown in Figure 8(c), was selected. The results presented and discussed from here on are all based on this model.
5.3. Numerical Stabilization. A geometrically nonlinear incremental analysis is carried out under displacement incrementation using the Newton-Raphson solution method. The equilibrium path of a wrinkled membrane includes many unstable branches, each corresponding to a localized snap-through with the formation of an additional wrinkle.

A very effective way of addressing the numerical singularities associated with instability is to switch from a quasistatic simulation to a transient dynamic analysis. In order to compute the first static equilibrium state after snapping has occurred, one would simulate the actual dynamic response of the structure as it snaps. This idea has been implemented in ABAQUS using the STABILIZE function. So, the STABILIZE function is activated for the postwrinkling analysis step in this paper. By assuming that the energy dissipated is a prescribed fraction of the strain energy during the first step, the default fictitious viscous forces are calculated on the basis of the model's response in the first increment of the analysis step. This fraction is called damping factor in ABAQUS; to achieve good accuracy, it is generally desirable to set the STABILIZE damping factor as low as the analysis can be convergent. The smallest STABILIZE damping factor used in the inflatable tube simulations was $2 \times 10^{-6}$ in this paper.

5.4. Numerical Simulation and Results. After defining the finite element mesh, type of elements, and material properties, a wrinkling analysis employing thin shell elements can be typically performed. Numerical simulation was conducted at air pressure values of 3000,4000 , and $5000 \mathrm{pa}$; the loaddeflection behavior of the ETFE inflatable beam adopting shell model is shown in Figure 9.

Mode jumps and the sequence of jumps are clearly shown in the load-deflection curves, as seen in Figure 9. The sudden transitions from one wrinkled shape to another were observed experimentally in [9]. Each change in the number of wrinkles is a mode jump in the postbuckling response of the membrane. Mode jumps have been explained in terms of the interaction of the branches of a set of bifurcation points adjacent to the critical load. But in our experiment, mode jumps were not caught and were not observed from the load-deflection curves shown in Figure 4.

Table 6 lists the comparison of maximum bearing force at internal pressure values of $3 \mathrm{KPa}, 4 \mathrm{KPa}$, and $5 \mathrm{Kpa}$ between experiment and results from shell model and also lists the force corresponding to the first jump point at internal pressure values of $3 \mathrm{KPa}, 4 \mathrm{KPa}$, and $5 \mathrm{Kpa}$; the errors between them and experimental results are shown in Table 6.

Seen from Table 6, the maximum forces obtained from shell model are about $4 \%$ to $8 \%$ greater than the experimental critical loads. The forces corresponding to the first jump point are more close to the experimental critical load than the maximum force from shell model. So, it is proposed in this paper that the force corresponding to the first jump point is considered as the critical bearing force when using nonlinear postbuckling method. The errors of the critical bearing force between experiment and shell model are about $2 \%$ to $3 \%$. 


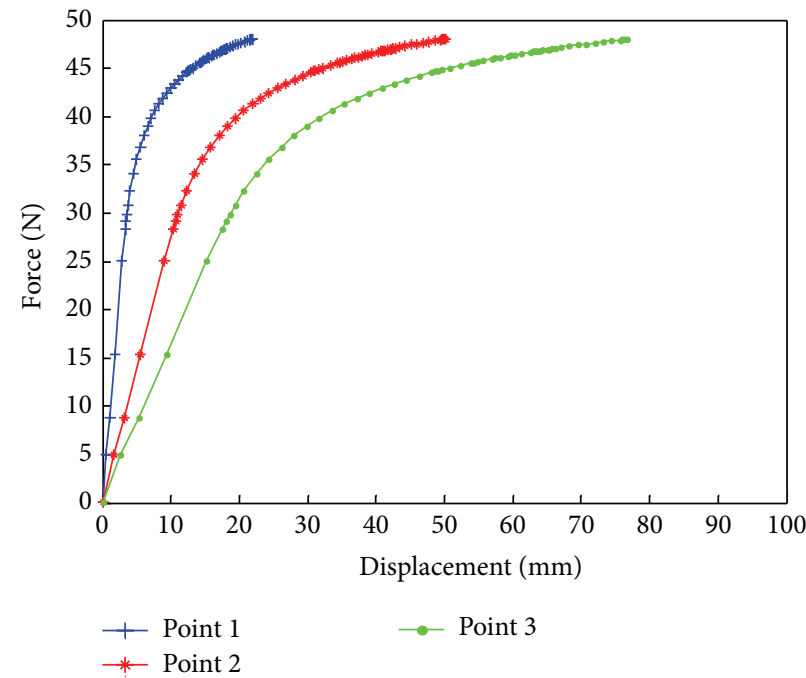

(a) Internal pressure $=3000 \mathrm{~Pa}$

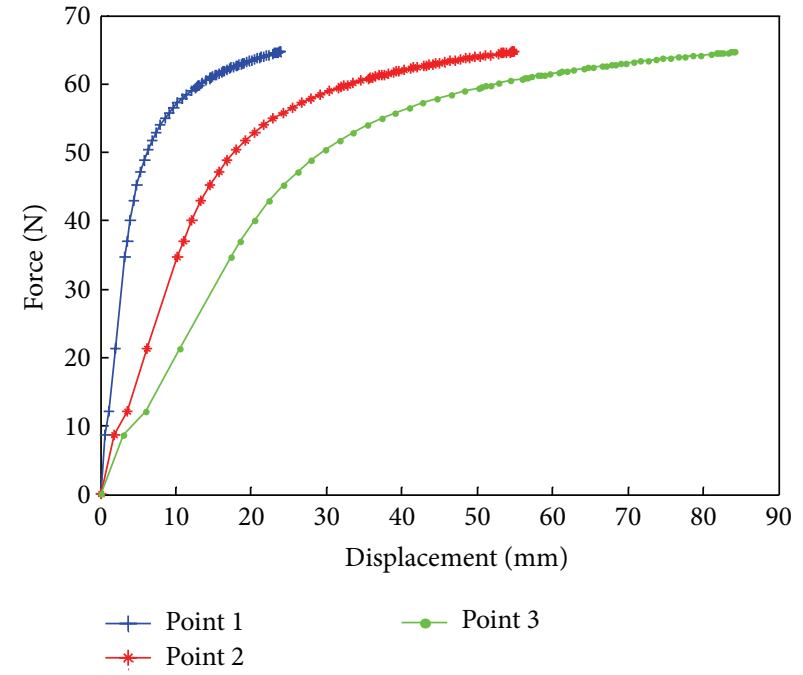

(b) Internal pressure $=4000 \mathrm{~Pa}$

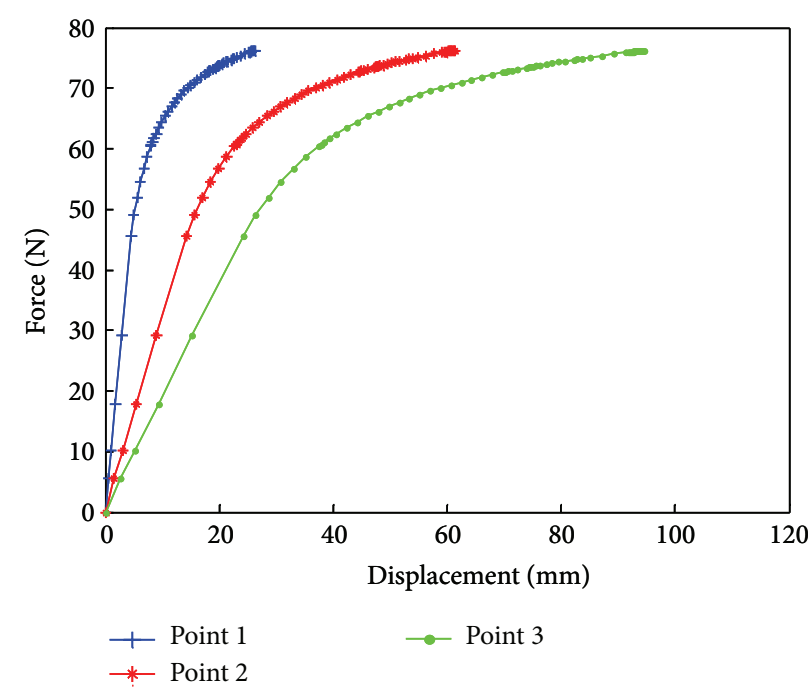

(c) Internal pressure $=5000 \mathrm{~Pa}$

FIGURE 7: Load-deflection behavior of the ETFE inflatable tube using tension field theory.

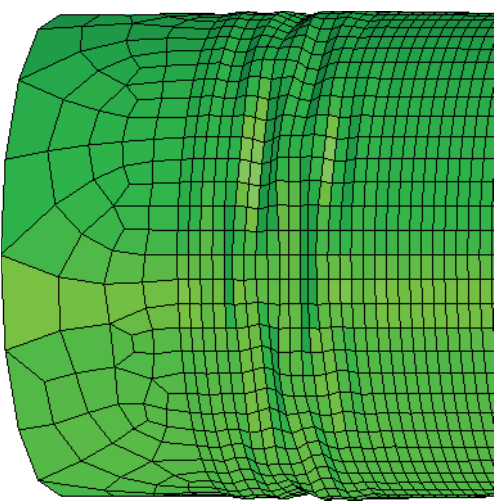

(a) Mesh aspect ratio $=40$

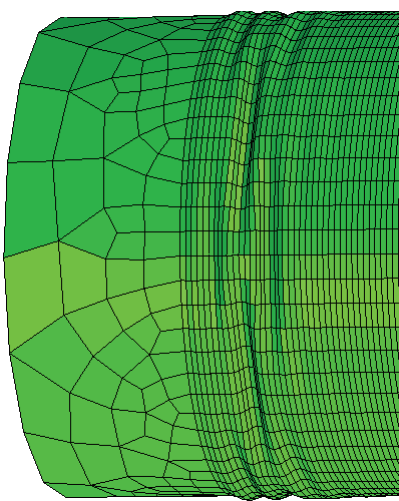

(b) Mesh aspect ratio $=20$

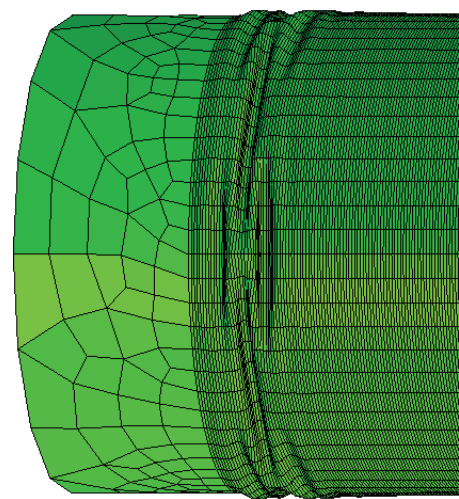

(c) Mesh aspect ratio $=10$

FIGURE 8: Wrinkles of the inflatable tube with different mesh density. 


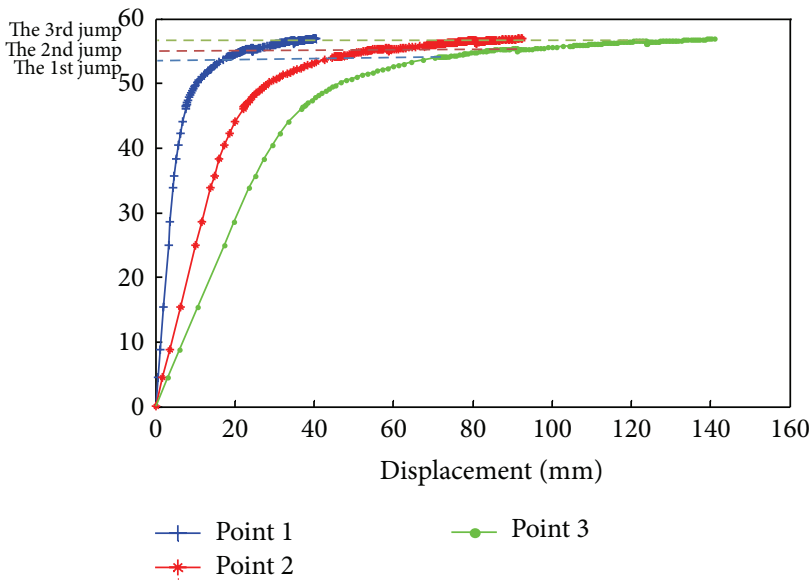

(a) Internal pressure $=3000 \mathrm{~Pa}$

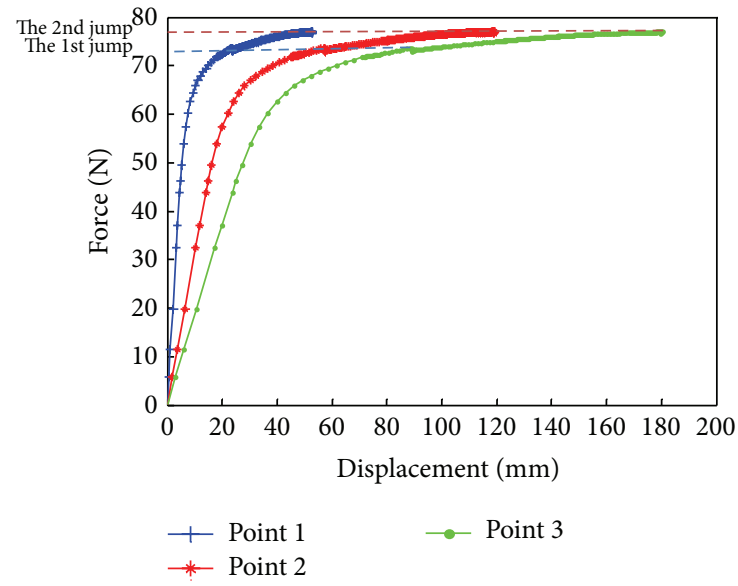

(b) Internal pressure $=4000 \mathrm{~Pa}$

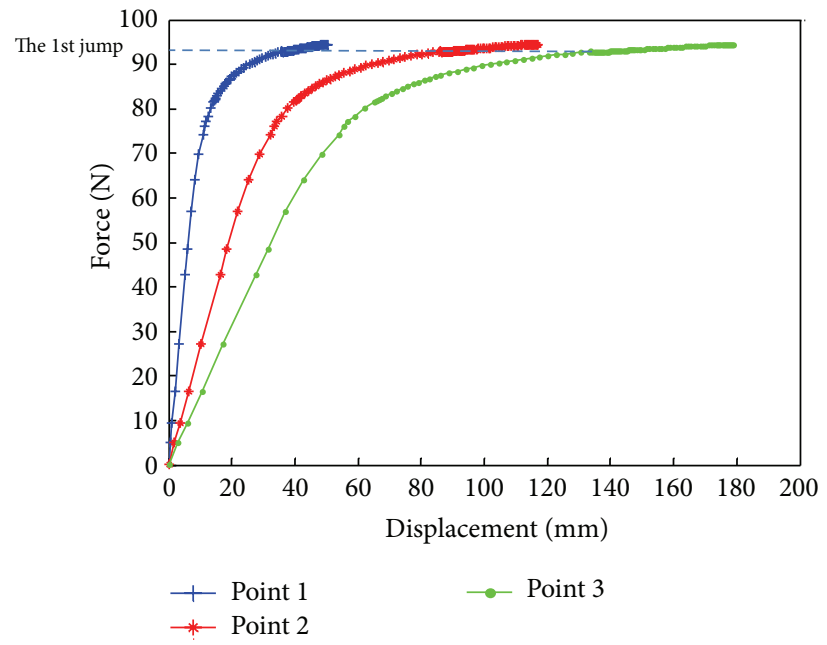

(c) Internal pressure $=5000 \mathrm{~Pa}$

FIGURE 9: Load-deflection behavior of the ETFE inflatable beam adopting shell model.

TABLE 2: Membrane properties.

\begin{tabular}{lcccc}
\hline Material & $\begin{array}{c}\text { Density } \\
\left(\mathrm{kg} / \mathrm{m}^{3}\right)\end{array}$ & $\begin{array}{c}\text { Young's modulus } \\
(\mathrm{MPa})\end{array}$ & Poisson's ratio & $\begin{array}{c}\text { Thickness } \\
(\mu \mathrm{m})\end{array}$ \\
\hline ETFE & 1700 & 680 & 0.31 & 250 \\
\hline
\end{tabular}

TABLE 3: Comparison of critical bearing capacity between experiment and IMP model.

\begin{tabular}{|c|c|c|c|}
\hline \multirow{2}{*}{ Internal pressure $(\mathrm{Pa})$} & \multicolumn{2}{|c|}{ Load bearing capacity $(\mathrm{N})$} & \multirow{2}{*}{ Error } \\
\hline & IMP model & Experiment & \\
\hline 3000 & 48 & 52.9 & $-9.4 \%$ \\
\hline 4000 & 64 & 72.5 & $-11.7 \%$ \\
\hline 5000 & 78 & 90.4 & $-13.7 \%$ \\
\hline
\end{tabular}

\section{Discussion and Conclusions}

A finite element simulation of a wrinkled inflatable tube has been carried out with the commercial finite element package ABAQUS, and a comparative study of two representative
TABLE 4: Sensitivity of inflatable tube to imperfection magnitudes.

\begin{tabular}{lcc}
\hline Internal pressure $(\mathrm{Pa})$ & $\phi_{1}, \ldots, \phi_{4}$ & Maximum bearing force $(\mathrm{N})$ \\
\hline \multirow{3}{*}{5000} & $10 \% t$ & 98.2 \\
& $25 \% t$ & 98 \\
& $100 \% t$ & 98 \\
\hline
\end{tabular}

TABLE 5: Dependence of maximum bearing force on mesh density.

\begin{tabular}{lcc}
\hline $\begin{array}{l}\text { Internal pressure } \\
(\mathrm{Pa})\end{array}$ & Mesh aspect ratio & $\begin{array}{c}\text { Maximum bearing force } \\
(\mathrm{N})\end{array}$ \\
\hline \multirow{3}{*}{5000} & 40 & 98 \\
& 20 & 94 \\
& 10 & 94 \\
\hline
\end{tabular}

theories, the bifurcation theory and the tension field theory, is carried out. The effects of imperfection and mesh density on the wrinkling behavior of an inflatable tube are investigated. The conclusions obtained in this study are summarized below. 
TABLE 6: Comparison of results between experiment and shell model.

\begin{tabular}{|c|c|c|c|c|c|}
\hline \multirow{2}{*}{ Internal pressure $(\mathrm{Pa})$} & \multicolumn{2}{|c|}{ The highest point } & \multicolumn{2}{|c|}{ First jump point } & \multirow{2}{*}{ Experimental critical load (N) } \\
\hline & Maximum force $(\mathrm{N})$ & Error & Buckling force $(\mathrm{N})$ & Error & \\
\hline 3000 & 57 & $7.5 \%$ & 54.2 & $2.26 \%$ & 52.9 \\
\hline 4000 & 76 & $4.8 \%$ & 72.2 & $0.4 \%$ & 72.5 \\
\hline 5000 & 94 & $3.9 \%$ & 92.5 & $2.32 \%$ & 90.4 \\
\hline
\end{tabular}

The critical bearing force obtained from engineering elastic beam theory is slightly less than the experimental critical load of the inflatable tube, but the load-deflection relationship cannot be provided by engineering elastic beam theory. So, the engineering elastic beam theory is often used to evaluate the critical load in the preliminary design of inflatable tubes.

The maximum bearing force of inflatable tube using tension field theory is about $13 \%$ lower than results from experiment, because tension field theory is considered as plate theory when it is applied to wrinkling analysis; the effect of relative bending stiffness on the strain energy stored in the wrinkled membrane is ignored. The wrinkled regions having some compressive stresses appear because the wave of wrinkles of the inflatable tube is constrained by the geometrical condition. Conventionally, tension field theory is not properly applied to these regions. Thus, the limitation of conventional tension field theory is quantitatively clarified.

The maximum bearing capacity obtained from geometrically nonlinear analysis is slightly larger than the experimental critical load of the inflatable tube, but the force corresponding to the first jump point coincides with the result from experiment.

\section{Conflict of Interests}

The authors declare that there is no conflict of interests regarding the publication of this paper.

\section{References}

[1] E. H. Mansfield, "Tension field theory," in Proceeding of the 12th International Congress of Applied Mechanics, pp. 305-320, Standford University, Stanford, Calif, USA, 1969.

[2] E. H. Mansfield, "Load transfer via a wrinkled membrane," Proceeding of the Royal Society A, vol. 316, no. 1525, pp. 269-289, 1970.

[3] A. C. Pipkin, "The relaxed energy density for isotropic elastic membranes," IMA Journal of Applied Mathematics, vol. 36, no. 1, pp. 85-99, 1986.

[4] R. K. Miller and J. M. Hedgepeth, "An algorithm for finite element analysis of partly wrinkled membranes," AIAA Journal, vol. 20, no. 12, pp. 1761-1763, 1982.

[5] R. K. Miller, J. M. Hedgepeth, V. I. Weingarten, P. Das, and S. Kahyai, "Finite element analysis of partly wrinkled membranes," Computers \& Structures, vol. 20, no. 1-3, pp. 631-639, 1985.

[6] A. L. Adler, Element approaches for static and dynamic analysis of partially wrinkled membrane structures [Ph.D. thesis], University of Colorado, Boulder, Colo, USA, 2000.
[7] Q. S. Yang, F. Tan, and X. F. Wang, "Loading and wrinkling analysis of membrane structures," Science China Technological Sciences, vol. 54, no. 10, pp. 2597-2604, 2011.

[8] Y. Tomita and A. Shindo, "Onset and growth of wrinkles in thin square plates subjected to diagonal tension," International Journal of Mechanical Sciences, vol. 30, no. 12, pp. 921-931, 1988.

[9] Y. W. Wong and S. Pellegrino, "Wrinkled membranes part I: experiment," Journal of Mechanics of Materials and Structures, vol. 1, no. 1, pp. 1-23, 2006.

[10] Y. W. Wong and S. Pellegrino, "Wrinkled membranes part II: analytical models," Journal of Mechanics of Materials and Structures, vol. 1, no. 1, pp. 25-59, 2006.

[11] Y. W. Wong and S. Pellegrino, "Wrinkled membranes III: numerical simulations," Journal of Mechanics of Materials and Structures, vol. 1, no. 1, pp. 63-95, 2006.

[12] A. Jarasjarungkiat, R. Wüchner, and K.-U. Bletzinger, "A wrinkling model based on material modification for isotropic and orthotropic membranes," Computer Methods in Applied Mechanics and Engineering, vol. 197, no. 6-8, pp. 773-788, 2008.

[13] A. Jarasjarungkiat, R. Wüchner, and K.-U. Bletzinger, "Efficient sub-grid scale modeling of membrane wrinkling by a projection method," Computer Methods in Applied Mechanics and Engineering, vol. 198, no. 9-12, pp. 1097-1116, 2009.

[14] C. G. Wang, H. F. Tan, X. W. Du, and Z. M. Wan, "Wrinkling prediction of rectangular shell-membrane under transverse in-plane displacement," International Journal of Solids and Structures, vol. 44, no. 20, pp. 6507-6516, 2007.

[15] H.-F. Tan, Z.-Y. Du, and C.-G. Wang, "Prediction on deflection of inflated beam," Journal of Harbin Institute of Technology, vol. 20, no. 1, pp. 8-11, 2013.

[16] T.-T. Nguyen, S. Ronel, M. Massenzio, E. Jacquelin, K. L. Apedo, and H. Phan-Dinh, "Numerical buckling analysis of an inflatable beam made of orthotropic technical textiles," ThinWalled Structures, vol. 72, pp. 61-75, 2013.

[17] W.-W. Xiao, W.-J. Chen, and G.-Y. Fu, "Wrinkle analysis of a space planar film reflect-array," Journal of Zhejiang University: Science A, vol. 12, no. 1, pp. 24-32, 2011.

[18] S. Timoshenko and K. S. Woinowsky, Theory of Plate and Shell, McGraw-Hill, New York, NY, USA, 1959.

[19] H.-J. Gao, W.-J. Chen, and G.-Y. Fu, "Structural design conception and analysis for the structural system of large flexible airship," Journal of Shanghai Jiaotong University (Science), vol. 15, no. 6, pp. 756-761, 2010.

[20] R. L. Comer and S. Levy, "Deflections of an inflated circularcylindrical cantilever beam," AIAA Journal, vol. 1, no. 7, pp. 1652-1655, 1963.

[21] M. Stein and J. M. Hedgepeth, "Analysis of partly wrinkled membranes," NASA Technical Notes D-813, 1961. 
[22] B. Zhao, Experiment study on material properties and doublelayer cushion structures of etfe foil [Ph.D. dissertation], Shanghai Jiao Tong University, Shanghai, China, 2012, (Chinese).

[23] W. J. Chen, L. Zhang, and D. X. Zhang, "Research and development of bi-axial tension tester and experiments on the mechanical properties of envelop fabrics," in Proceedings of the International Conference on Textile Composites and Inflatable Structures (STRUCTURAL MEMBRANES '11), Barcelona, Spain, 2011. 

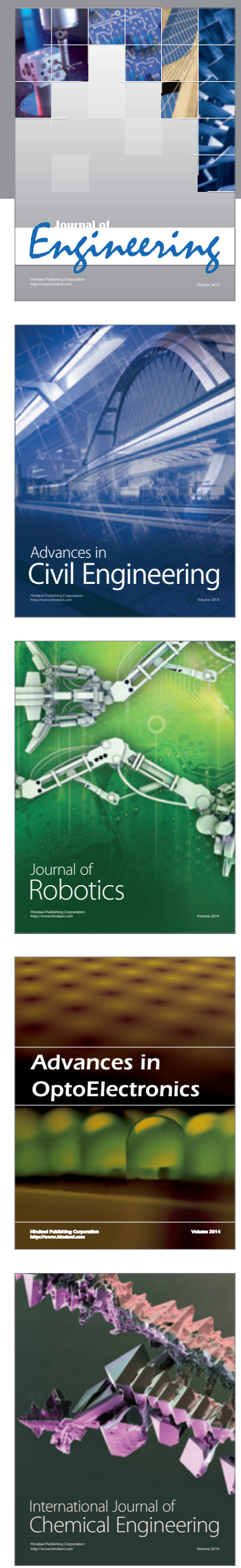

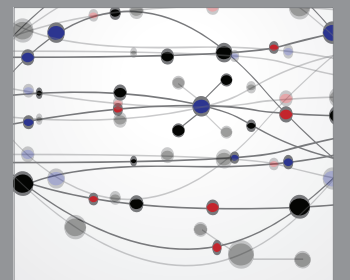

The Scientific World Journal
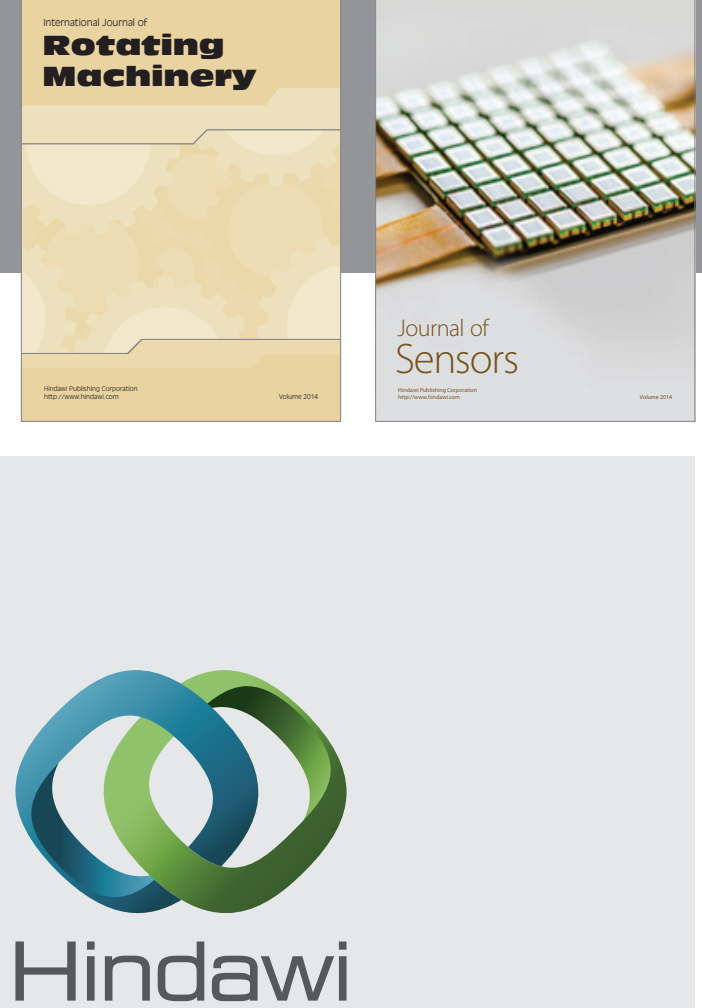

Submit your manuscripts at http://www.hindawi.com
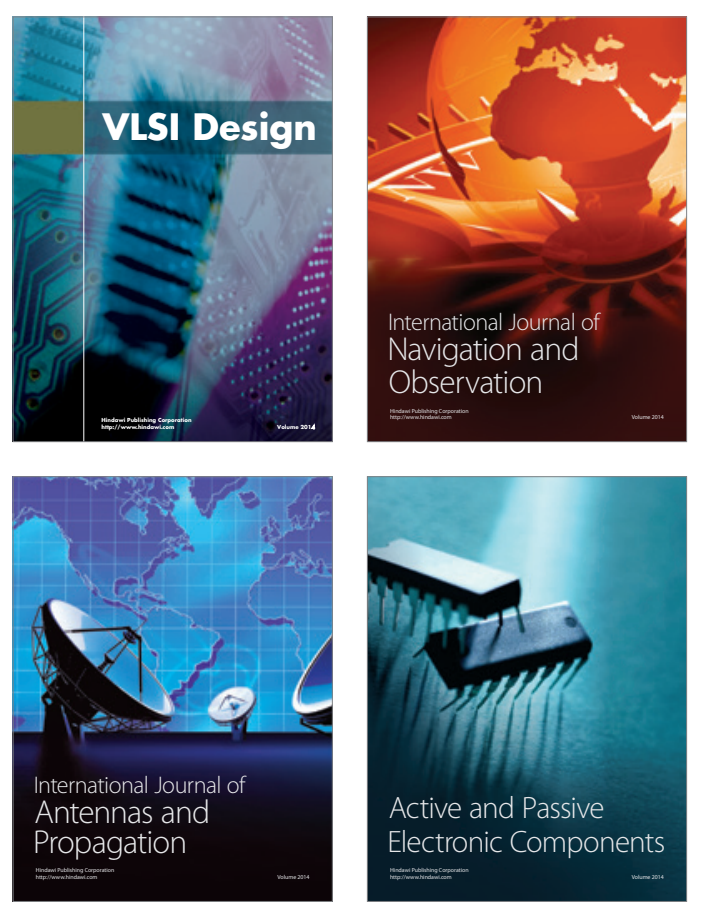
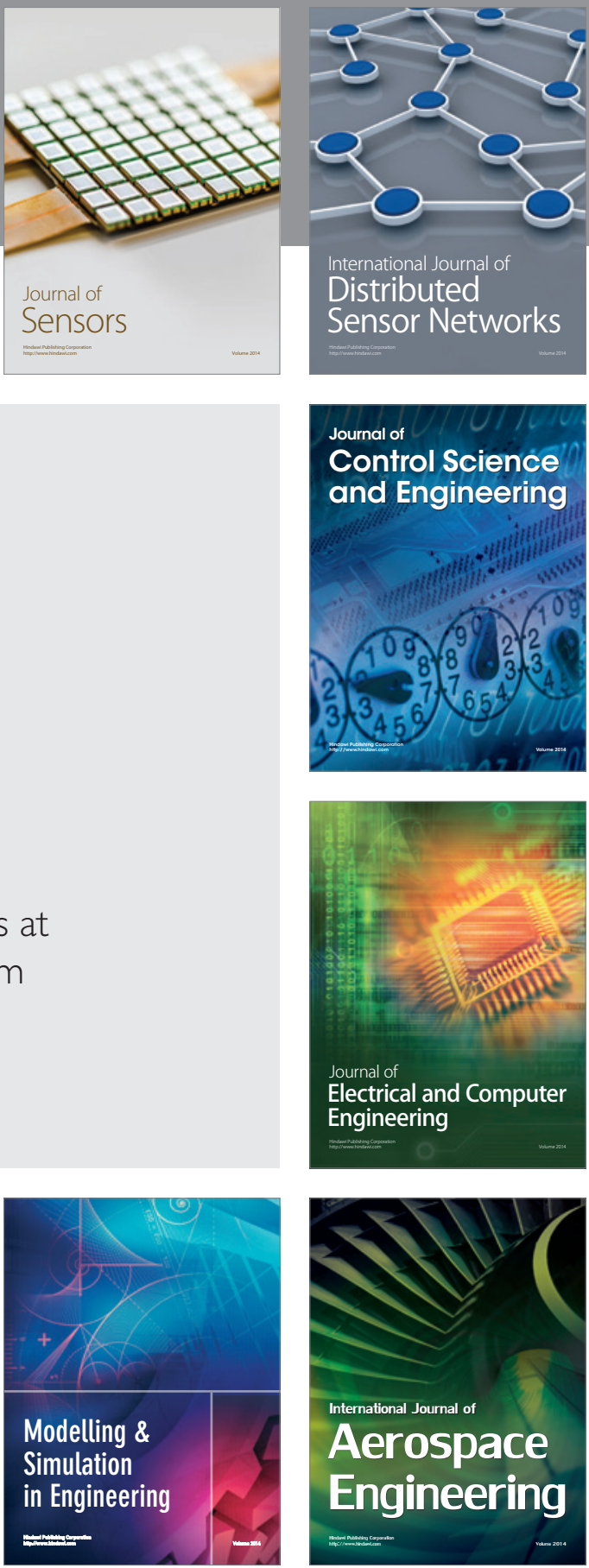

Journal of

Control Science

and Engineering
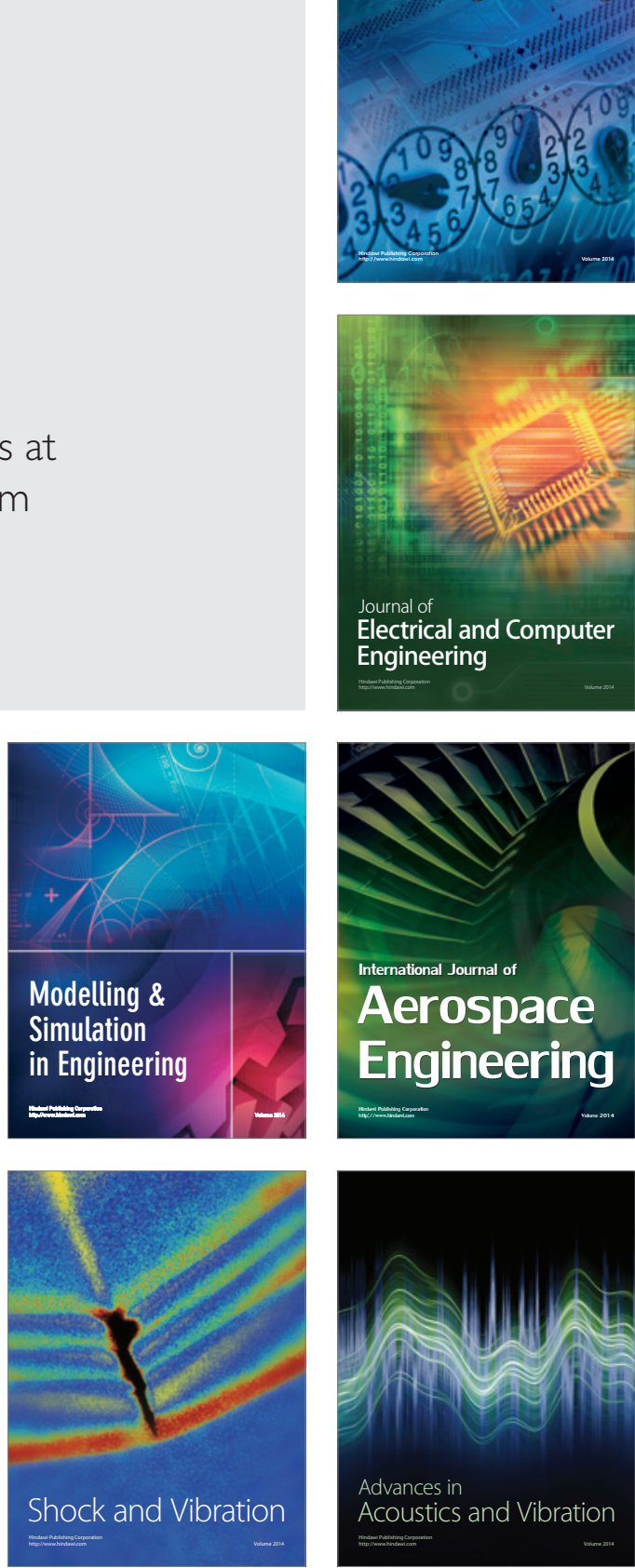